\title{
158. Pharmakologische Untersuchungen über ",Senso,“ eine chinesische Droge aus abgetrocknetem Hautsekret der Kröte.
}

\section{Mitteilung.}

Von Yoshito KoBaYashI.

Pharmakologisches Institut, Tokyo Kaiserliche Universität. (Rec. Oct. 20, 1933. Comm. by K. MiurA, M.I.A., Nov. 13, 1933.)

Über die chemischen und pharmakologischen Untersuchungen von „Senso " liegen bis jetzt Arbeiten von Shimizu, Kodama und Kotake vor, und über solche des Hautsekretes der Kröte von Faust, Wieland, Abel und Kotake. Die Resultate der Autoren über die daraus gewonnenen wirksamen Bestandteile lauten jedoch sehr verschieden.

Kondo und Ikawa gelang es nun, aus „Senso “ das $\psi$-Bufotalin und aus dem Hautdrüsensekret der chinesischen Kröte, dem Rohmaterial von Senso, das $\psi$-Bufotoxin zu isolieren, und daraus verschiedene Derivate herzustellen. Wir haben daher unternommen, diese Präparate pharmakologisch an Kalt- und Warmblütern zu untersuchen und gefunden, dass das $\psi$-Bufotalin-Bromid das beste Präparat ist.

In der vorliegenden Mitteilung werden hauptsächlich Untersuchungen erwähnt, die an isolierten Froschherzen mit $\psi$-Bufotalin und seinen Derivaten angestellt wurden. Die Resultate weiterer Untersuchungen sind den späteren Mitteilungen vorbehalten.

$\psi$-Bufotalin stellt eine fein krystallinische Substanz von Fp 145-146 mit der Formel: $\mathrm{C}_{25} \mathrm{H}_{36} \mathrm{O}_{6}$ dar. (Kondo und Ikawa.)

Die Substanz zeigte nach dem Perfusionsversuche des isolierten Froschherzens eine digitalisähnliche cardiotonische Wirkung; selbst bei der Verdünnung von 1:1000000000 machte sich eine leichte Vergrösserung der Amplitude der Kammerschläge bemerkbar, während bei der Verdünnung von 1:10000000 schon die Vergiftungserscheinungen eintraten. Nach der Hatcherschen Wertbestimmung betrug die tödliche Dosis pro Kilogramm Katze $0.25 \mathrm{mg}$.

Des-Acetyl- $\psi$-Bufotalin, $\mathrm{C}_{24} \mathrm{H}_{34} \mathrm{O}_{5}$, Zers. bei ca. $130^{\circ}$, krystallinisch.

Die aus dem Acetoxyl hervorgehende Hydroxylgruppe tautomerisiert zur Ketogruppe, wie $>\mathrm{C}: \underset{1}{\mathrm{C}} \cdot \mathrm{O} \cdot \mathrm{CO} \cdot \mathrm{CH}_{3} \rightarrow>\mathrm{C}: \underset{!}{\mathrm{C}} \cdot \mathrm{OH} \rightarrow>\underset{1}{\mathrm{CH}} \cdot \mathrm{C}: \mathrm{O}$ (Kondo und Ikawa). Die Substanz erweist sich weniger wirksam als 


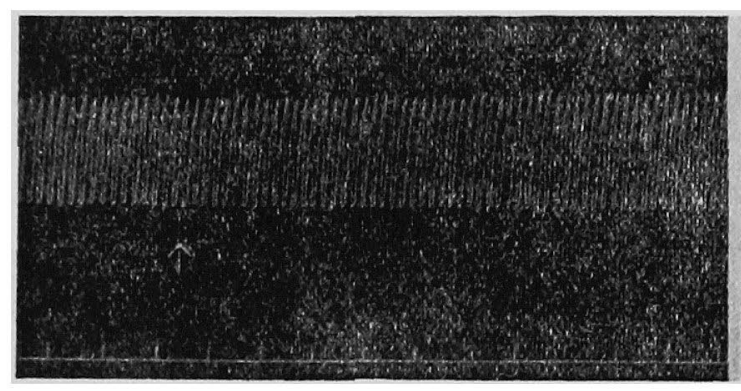

I

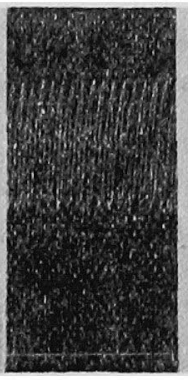

II

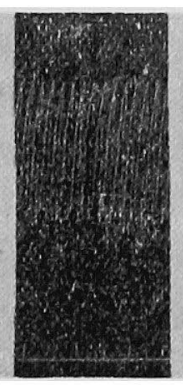

III

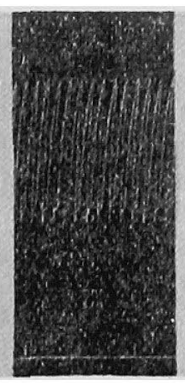

IV

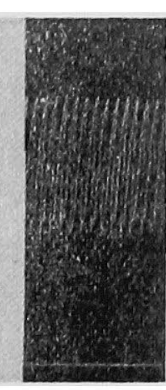

$\mathrm{V}$

Fig. 1. Isoliertes Froschherz wird von V cava mit Ringerscher Lösung durchströmt. I : $\uparrow$ Beginn des Einlaufes mit $\psi$-Bufotalinlösung $1: 1000000000$; II : 2 Min.; III : 5 Min.; IV : 7 Min.; V : 10 Min. nach $\psi$-Bufotalin. Zeit: 6 Sek.

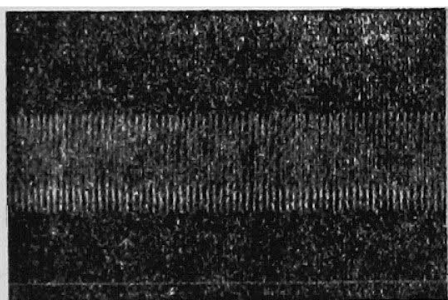

I

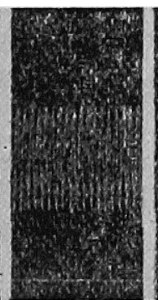

II

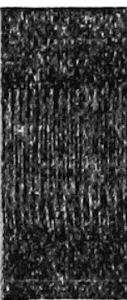

III

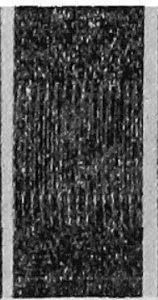

IV

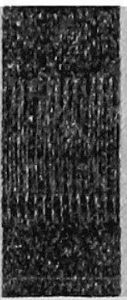

V

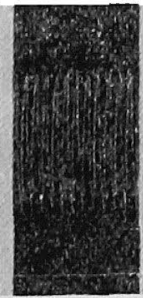

VI

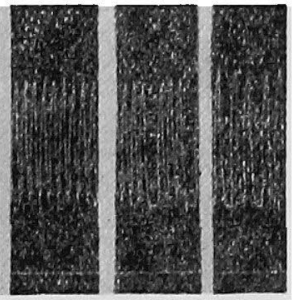

VII VIII IX

Fig. 2. Isoliertes Froschherz. I: $\uparrow$ Beginn des Einlaufes mit $\psi$-Bufotalinlösung $1: 10000000$; II : 2 Min.; III : 5 Min.; IV : 7 Min. ; V : 10 Min.; VI : 15 Min.; VII : 20 Min.; VIII : 22 Min.; IX : 25 Min. nach $\psi$-Bufotalin. Zeit: 6 Sek.

Bufotalin; sie zeigt bei der Verdünnung von 1:30000000 minimale Wirkung auf das Herz; es ist aber bemerkenswert, dass selbst bei der Verdünnung von 1:1000000 keine Vergiftungserscheinung auftritt, was bei Bufotalin stets der Fall ist. Hieraus kann man schliessen, dass die in der Enolform sich befindliche Acetoxylgruppe in Bezug auf die Herzwirkung eine grosse Rolle spielt.

$\psi$-Bufotalin-Bromid, $\mathrm{C}_{25} \mathrm{H}_{35} \mathrm{O}_{5} \mathrm{Br}, \mathrm{Fp} 100-114^{\circ}$, krystallinisch.

Es wurde hier genauer studiert, inwieweit die cardiotonische Wirkung des Bufotalins durch die Einführung des Broms beeinflusst wird. Dabei konnte man zum grossen Interesse festgestellten, dass die cardiotonische Wirkung auf das isolierte Froschherz über $10000 \mathrm{mal}$ stärker ist als Bufotalin, während die Giftigkeit dieser Verbindung mit Brom dem Bufotalin beträchtlich nachsteht. Selbst bei der Verdünnung von 1:1000000000000000 lässt sich eine mässige Vergrösserung der Kammerkontraktionen nachweisen; die toxische Erscheinung tritt dagegen bei einer Verdünnung von 1:1000 000 noch nicht auf, erst bei 
einer solchen von 1:10000-50000 ist eine solche bemerkbar. Nach der Hatcherschen Wertbestimmung beträgt die tötliche Dosis pro Kilogramm Katze über $19 \mathrm{mg}$.

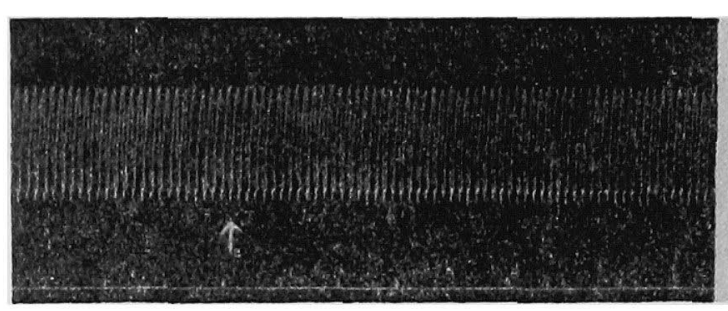

I

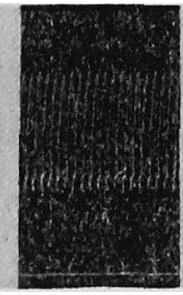

II

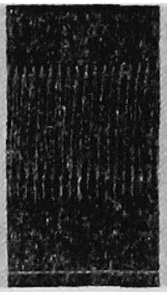

III

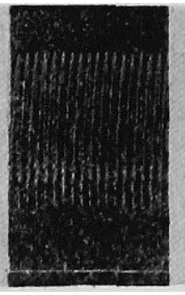

IV

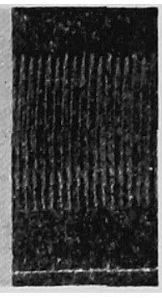

$\mathrm{V}$

Fig. 3. Isoliertes Froschherz. I: $\uparrow$ Beginn des Einlaufes mit 4 -Bufotalinbromidlösung 1:1000 000000000000 ; II : 2 Min.; III : 5 Min.; IV : 10 Min. ; V : 12 Min. nach $\psi$-Bufotalinbromid. Zeit: 6 Sek.

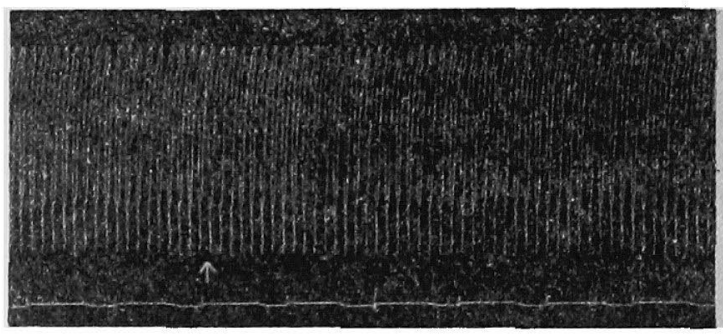

I

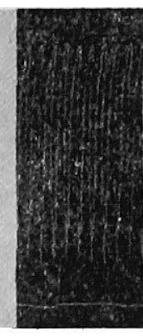

II

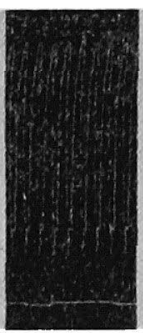

III

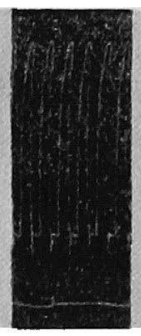

IV

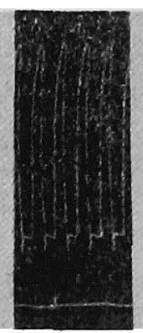

V

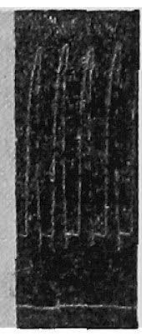

VI

Fig. 4. Isoliertes Froschherz. I: $\uparrow$ Beginn des Einlaufes mit $\psi$-Bufotalinbromidlösung $1: 50000$; II : 5 Min.; III : 10 Min.; IV : 20 Min.; V : 25 Min.; VI : 30 Min. nach $\psi$-Bufotalinbromid. Zeit: 10 Sek.

Des-Acetyl- $\psi$-Bufotalinchlorid, $\mathrm{C}_{24} \mathrm{H}_{33} \mathrm{O}_{4} \mathrm{Cl}$, Zers. bei $140-145^{\circ}$ krystallinisch.

Bei der Verdünnung von 1:5000000000 lässt sich eine mässige cardiale Wirkung nachweisen, aber selbst bei der Verdünnung von 1:1000 000 treten die Vergiftungserscheinungen nie auf. Hieraus geht hervor, dass die Verbindungen, deren eigentliches tertiäres Hydroxyl mit Halogen ersetzt ist, cardial wirksamer werden als die Grundsubstanz, ohne Zunahme der Giftigkeit.

Monoacetyl- $\psi$-Bufotalin, $\mathrm{C}_{23} \mathrm{H}_{38} \mathrm{O}_{7}$, Fp ca. $120^{\circ}$, krystallinisch.

Die cardiotonische Wirkung erweist sich bedeutend schwächer als Bufotalin. Die minimale wirksame Konzentration beträgt ca. $1: 10000000$.

Monoacetyl-Desacetyl- $\psi$-Bufotalinchlorid, $\mathrm{C}_{26} \mathrm{H}_{35} \mathrm{O}_{5} \mathrm{Cl}$, Zers. bei 160 $165^{\circ}$, krystallinisch. 
No. 9.] Pharmakologische Untersuchungen über „, Senso,“ eine chinesische Droge. 555

Die Substanz ist weniger wirksam als die Muttersubstanz, aber gleichzeitig fast gleich giftig $(1: 1000000)$.

Anhydro- $\psi$-Bufotalinchlorid, $\mathrm{C}_{26} \mathrm{H}_{33} \mathrm{O}_{4} \mathrm{Cl}, \mathrm{Fp}$ ca. $120-130^{\circ}$, krystallinisch.

Die wirksame Verdünnung ist $1: 100000000$; es ist also weniger wirksam als Bufotalin.

$\psi$-Bufotalon, $\mathrm{C}_{26} \mathrm{H}_{34} \mathrm{O}_{6}$, Fp ca. $135^{\circ}$, krgstallinisch.

Die Substanz bleibt in ihrer Herzwirkung fast unverändert im Vergleich mit Bufotalin.

$\psi$-Bufotoxin, $\mathrm{C}_{40} \mathrm{H}_{62} \mathrm{O}_{11} \mathrm{~N}_{4}$, krystallinisch.

Die Substanz ist nur wenig in "Senso " vorhanden, dagegen reichlich im frischen Hautsekrete der chinesischen Kröte. Ihre Wirkung auf das Herz ist etwas schwächer als Bufotalin; bei der Verdünnung von 1:500 000000 lässt sich eine geringe Zunahme der Systole nachweisen.

Des-Acetyl- $\psi$-Bufotalinsäure, $\mathrm{C}_{24} \mathrm{H}_{36} \mathrm{O}_{6}$ und Des-Acetyl- $\psi$-Bufotalonsäure $\mathrm{C}_{24} \mathrm{H}_{34} \mathrm{O}_{6}$.

Beide Bubstanzen wirken mehr oder weniger auf das Herz. Bei der Verdünnung von $1: 20000-40000$ zeigt sich zuerst eine Verstärkung der Kammerkontraktion, die bei weiterer Verdünnung nicht mehr in Erscheinung tritt. Die Metylester haben eine etwas stärkere Wirkung auf das Herz als die Säure selbst.

Tetrahydro- $\psi$-Bufotalin, $\mathrm{C}_{26} \mathrm{H}_{40} \mathrm{O}_{6}$.

Es gibt keine Doppelbindung im Molekül. Am Herzen wirkt sie auffallend schwach, erst bei der Konzentration von 1:20000 tritt die cardiotonische Wirkung in geringem Grade auf. Die Einführung des Halogens in das Tetrahydrobufotalin verursacht keine Verstärkung der Herzwirkung mehr.

\section{Literatur.}

Kondo und Ikawa: Journ. of Pharmaceutic Soc. of Japan, 611 (1933), 1 ; 614 (1933), 62. 